\title{
DESIGNOR: A Decision Support Procedure for Industrial Product Design
}

\author{
Jean-Marie Choffray. Ecole Superieure des Sciences Economiques et \\ Commerciales, Paris \\ Gary L. Lilien. The Pennsylvania State Universiti. L'niversity Park
}

\begin{abstract}
This paper presents a model-based methodology called DESIGNOR to suppor design decisions for new industrial products. Models and measurements are developed to assess the relationship between product features and market potential. The methodology: incorporates a measurement survey for model calibration and leads to empirical tradeoff curves between design dimensions. The approach handles discrete as well as continuous teatures and can be incorporated into a procedure aimed at finding a profit maximizing design. It can be used to support decisions concerning product design as well as for product line expansion. The approach provides a consistent way of evaluating market potential for new industrial products and presents important advantages over other product design methodologies.
\end{abstract}

\section{Introduction}

The long-term health of many industrial enterprises is closely tied to their ability to continue to produce successful new products. But as markets become more competitive, risks increase and failure costs become more severe [9]. Traditional approaches to the design and marketing of new products are being supplemented by operational decision-support systems, blending managerial experience with management science methods [7].

As with most developments in marketing, this trend is most pronounced in the consumer goods area. Urban and Hauser [11] give a comprehensive review of these developments. In the area of industrial product development particularly for capital equipment, many of these approaches do not apply. The existence of organizational constraints as well as the multiperson nature of the industrial adoption process requires substantial methodological modifications [3].

The purpose of this paper is to develop and describe a procedure called DESIGNOR aimed at assessing the market potential for a new industrial

\footnotetext{
Address correspondence to Gary L. Lilien, College of Business Administratiun, The Pennsylvania State University, University Park, PA 16802.
} 
product as a function of the product's design features. Tradeoffs can be inferred from the DESIGNOR model, allowing different designs with the same market potential to be explicitly examined. Assumptions about costs for product features can be combined in a design optimization model. Implementation of the procedure is illustrated and directions for future research are discussed.

\section{Modeling Industrial Market Response}

Market demand and market potential are two critical figures for new product planning but are often used loosely.

Market demand is the total volume of a product sold under a defined marketing program [6]. In contrast, market potential is the amount that would be sold if the marketing effort were arbitrarily large. It is the limit that market demand would tend to, if marketing conditions were most favorable. For a new industrial product, then, market potential comprises the needs of every company that can use the product and for which it meets purchasing requirements.

The current theory of organizational buying behavior suggests that new product adoptions may be viewed as a sequential elimination process 113,31 . The setting of purchase specifications is one of the major steps in the purchasing process, screening out purchase alternatives that do not meet the firm's requirements $[10,4]$. Acceptable alternatives are then further assessed in a process that leads to the organizational choice.

Figure I characterizes this process and relates it to the concepts of market potential and market demand. As a first step, the new product design is assessed against purchase specifications within firms that comprise its target market. This yields its market potential. The evaluation and choice process that follows compares the new product with other acceptable alternatives to give a demand evaluation.

Formally, this is equivalent to the following decomposition of product demand. Define $X=\left(X_{1}, \cdots, X_{j}\right)$ as the product design under evaluation where $X_{1}, \cdots, X_{\text {, }}$ are specific features. We can write a demand forecast for $X$ as

Demand Forecast $X=($ Number of target firms aware of Product $X)$

$$
\begin{aligned}
& X \text { (Fraction of firms that find Product } X \\
& \text { acceptable) } \\
& \times \text { (Fraction of firms that prefer Product } X \text { to } \\
& \text { other acceptable offerings). }
\end{aligned}
$$




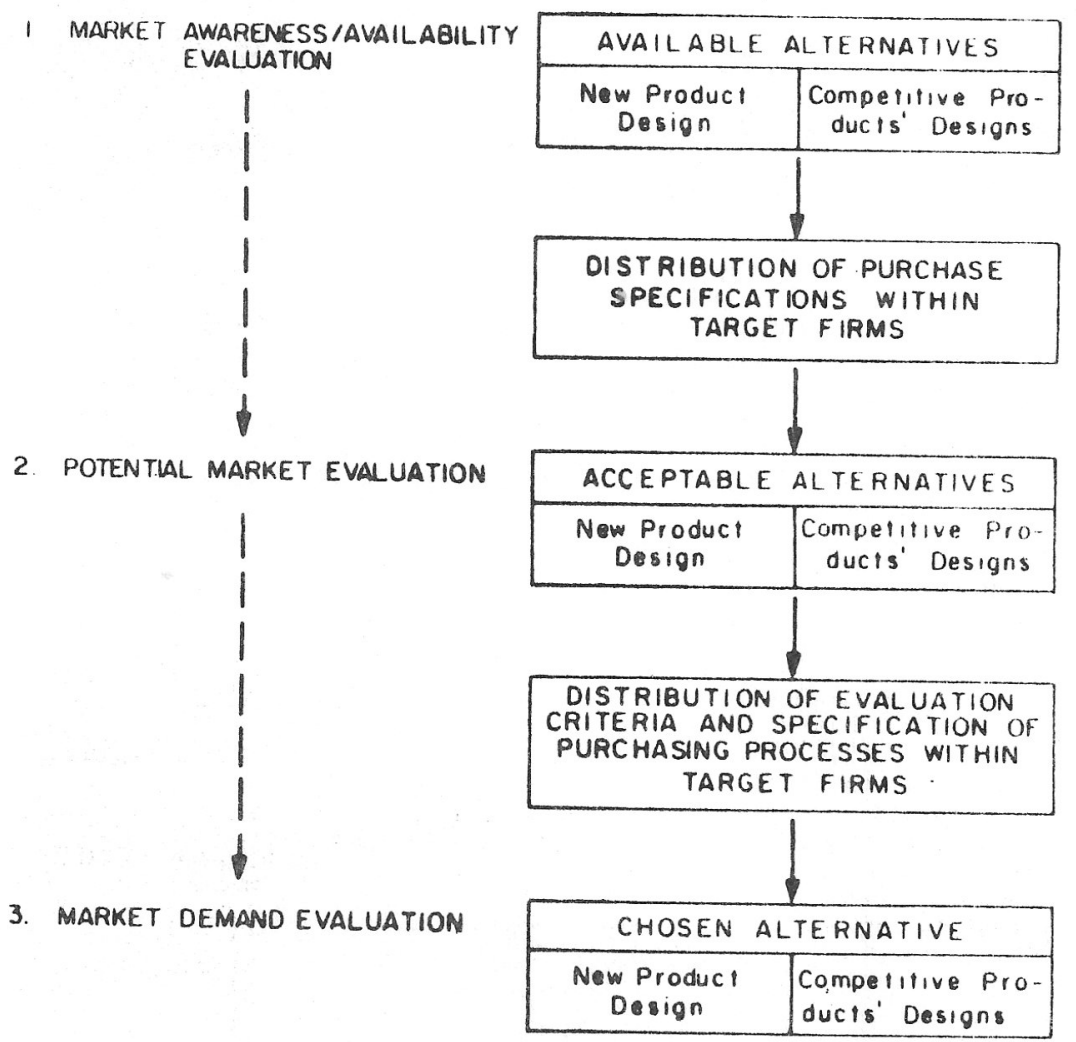

FIGURE 1: Flow model of organizational elimination of product alternatives.

The DESIGNOR model focuses on the second step, the interaction between product design and market potential. We call criteria used to reduce an organization's choice set to acceptable alternatives "need specification dimensions." Translated into a marketer's perspective, they are product design dimensions. This concept is consistent with the notion of dimension of merit proposed by Von Hippel [12].

Need specification dimensions are normally of three types:

- Boundary specification dimensions. The firm specifies an extremum (minimum or maximum value) beyond which a product is rejected as infeasible. A potential customer for a lathe may require the warranty period to be of at least 18 months and the initial cost to be under $\$ 15,000$.

- Range specification dimensions. Products must fall into a specific range along the dimension considered. Production tolerance range's are 

of this nature when the product purchased is to be incorporated in
another product or process.

- Discrete specification dimensions. Products must incorporate specific teatures (e.g., automatic feed on an office copier).

\section{Operationalizing the DESIGNOR Methodology}

The development and calibration of a model to relate industrial market acceptance 10 design decisions requires that we consider (wo sources of
heterogeneity:

- Specification dimension heterogeneity. Organizations may differ in their dimensions to set requirements.

- Requirements heterogeneity. Organizations sharing the same set of specification dimensions may have different requirements along those
dimensions.

To account for these sources of heterogeneity the DESIGNOR methodology follows the procedure outlined in Table 1.

Measurement Step I in the methodology identifies need specification dimensions and measures purchase requirements along these dimensions. The development of questions to measure these variables follows a two-step procedure.

First, a series of open-ended interviews are conducted with members of the buying center in a few (5-10) target companies. These interviews

Table 1: Development of the Market Acceptance Model

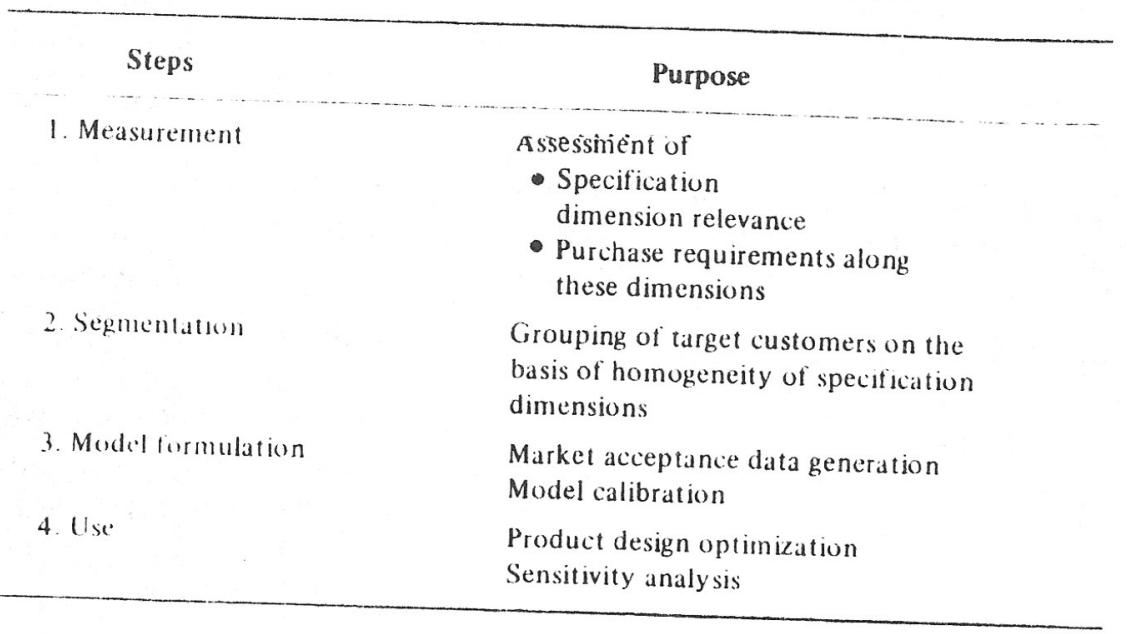


identify an exhaustive set of specification dimensions for profucts in the class. Content analysis of these interviews leads to prototype survey questions for assessing organizations ${ }^{\circ}$ requirements.

Second, these questions are administered to a sample of target companies. Where appropriate, several individuals in the organization are surveyed. To reduce individual response bias, respondents may use any information sources in their organization to provide their answer. A formal procedure to account for individual response bias is available in Choffray and Lilien [3].

Let $\underline{X}\left(X_{j}, j=1 \cdots \cdot \mathcal{l}\right)$ denote the exhaustive set of specification dimensions. An individual company $i$ may use only a subset of these dimensions for product elimination. Call this subset $X$. Hence $X, \cdots X_{1}$ $\cup \cdots \cup X_{i}, l<J$. Company $i$ 's specific requirements along the se dimensions are written $\mathcal{L}_{i}$, where $x_{i k}$ might be the maximum feasible value along dimension $k$ for company $i$.

Segmentation The next step requires that we group target customers on the basis of common specification dimensions. Agglomerative clustering is used for this purpose. Methods for performing such analysis are discussed in Anderberg [1] and Choffray and Lilien [3].

Let $X^{(n)}, s=1 \cdots S$ denote the subset of specification dimensions shared by companies belonging to segment $s$. Hence $X=X^{(1)}$ $\cup X^{(2)} \ldots \cup \underline{X}^{(s)}$. Relative segment sizes are denoted $w_{s}, s=1, \cdots, s$,
with $\sum_{s=1}^{s} w_{s}=1$.

Development of a Segment Acceptance Model Consider segment $s$, comprising target firms that share specification dimensions $X^{\prime \prime \prime}$. The purpose of the segment acceptance model is to relate design characteristic requirements to the acceptance rate or fraction of firms that will find it acceptable. Let $y=\left(y_{1}, \cdots, y_{j}\right)$ denote a product design corresponding to the exhaustive set of product features.

The following form provides a reasonable approximation of the conjunctive elimination process nutlined earlier. We call it the

$$
P(\underline{y})=\alpha_{0} \prod_{k=1}^{K}\left[p_{k}\left(y_{k}\right)\right]^{\alpha_{k}}
$$

where

$\begin{aligned} P(y) & = \\ & \text { tics } y \\ = & \text { partial acceptance rate for design characteris- } \\ p_{k}\left(y_{k}\right) & \text { tic } y_{k}\end{aligned}$ 


$$
\begin{aligned}
\alpha_{h}, k=0, \ldots, K= & \text { parameters to be estimated. } \\
= & \text { number of specification dimensions for the } \\
& \text { segment. }
\end{aligned}
$$

The partial acceptance rate here is a function of the corresponding design characteristics $\left\{y_{k}\right\}$. The form of this function varies according to the nature of the specification dimension, as described below.

Boundary Specification Dimensions A boundary specification dimension takes the form of either a minimum (such as warranty) or a maximum (such as price) value condition to be satisfied by the product.

If dimension $k$ corresponds to a minimum requirement, the observed partial acceptance rate for design characteristic $y$ is given by

$$
\pi_{k}\left(y_{k}\right)=\int_{0}^{y_{k}} f_{X_{k}}(x) d x=F_{X_{k}}\left(y_{k}\right)
$$

where $f_{x}$ refers to the observed distribution (frequency) of the individual specification dimension limit $x$.

In the same way, if dimension $k$ corresponds to a maximum requirement, the observed partial acceptance rate is given by

$$
\pi_{k}\left(y_{k}\right)=1-F_{X_{k}}\left(y_{k}\right) .
$$

Because of sampling variation, the actual segment partial acceptance rate $\left(p_{k}\right)$ may differ from the observed acceptance rate [2]. For our purposes we assume here that $p_{k}\left(y_{k}\right)$ can be approximated adequately by $\pi_{k}\left(y_{k}\right)$.

Range Specification Dimensions Products acceptable for a range specification dimension must fall within a given range for each target firm. Variations may exist across firms, both in the upper and lower bounds.

Let $X_{h}$ be the design dimension. Here we let $x_{k}$ be the lower range limit (with associated $f_{\lambda_{k}}, F_{x_{k}}$ ) and $r_{k}$ be the range length (with associated $f_{R_{k}}$, $F_{k_{k}}$. Then the partial acceptance rate for design characteristic $y_{k}$ is

$$
\begin{aligned}
p_{k}\left(y_{k}\right) & =\operatorname{Prob}\left(x_{k}<y_{k}<x_{k}+r_{k}\right) \\
& =\int_{0}^{y_{k}} \int_{y_{k} x_{k}}^{\infty} f_{X_{k} R_{k}}(x, r) d x d r \\
& =\int_{0}^{y_{k}} f_{X_{k}}\left(x_{k}\right)\left(1-F_{R_{k}}\left(y_{k}-x_{k}\right)\right) d x
\end{aligned}
$$

if $X_{h}, R_{h}$ are independent. 
Discrete Specification Dimensions The $0-1$ nature of discrete specification dimensions suggests that if $x_{k}$ is a discrete dimension, $y_{k}$ 0 or 1. If $y_{k}=1$, then $p_{k}\left(y_{k}\right)=$ proportion of the population that requires $y_{h}$.

Model Calibration For calibration purposes, note that the segment response model can be linearized easily:

$$
\ln [P(y)]=\ln \left(\alpha_{0}\right)+\sum_{k=1}^{K} \alpha_{k} \ln \left[p_{k}\left(y_{k}\right)\right]
$$

Parameters can be assessed by weighted least squares methods where the weights reflect the increase in the variance of $P(y)$ as $P$ comes closer to 0 or 1. Alternatively, maximum likelihood procedures might he used.

Observations needed for calibration purposes are obtained by sim. ulation. First, we randomly generate a number, $m$, of system designs $\left(y_{m k}\right) k=1, \cdots, n, m=1, \cdots, M$, where "randomly" means in accordance with the joint empirical distribution of critical acceptance levels. This forces observations to be obtained in the areas of maximum sensitivity and is related to the principle of sampling proportional to the standard deviation within each stratum. Second, for each design generated, we compute the observed partial acceptance rates using forms derived above and the joint acceptance rate.

Market Potential, Market Demand, and Product Design To assess the total market potential as a function of product design, we aggregate segment acceptance functions as follows:

$$
P(y)=\sum_{s=1}^{S} w_{s} P_{s}(\underline{y})
$$

where

$w_{s} \quad=$ fraction of the market in that segment

$P_{s}(\underline{y})=$ market acceptance function for segment $s$.

The impact of changes in product design $y$ can be assessed directly by the equation above. If in addition we know 1) the size of the target market for product $y, 2$ ) the unit cost of producing design $y$, and 3 ) the market share the firm could expect in the segment, a profit function can be constructed and a design developed to maximize profit. Note that owing to production economies, a sales forecast in terms of market share is needed to develop unit costs. In addition, market share as well as present potential will be functions of product design in general. 
A major use of the total market potential function may not be for product design optimization but, rather, for cost/benefit analysis of a product line extension strategy. This use is illustrated in the second example below.

\section{Case Examples}

New Product Design: Frozen Meals This example is from a study performed in France for a food-processing company. The product investigated is a new frozen complete meal, ready to serve, sold exclusively to restaurants.

A descriptive analysis of the purchasing decision process in the target market was performed and led to identification of the following
specification dimensions:

\begin{tabular}{ll}
\hline Specification Dimensions & Design Features \\
\hline Price (maximum) & French francs per meal \\
Freezer shelf life (minimum) & Months/packaging \\
Type of meat & Fish, Charolais beef, duck \\
Type of potato & Potato croquettes, Dauphinois gratin \\
\hline
\end{tabular}

Short-term production constraints prevented this firm from considering developing more than one product over the next 12 months. In view of the impact that the penetration achieved during that time would have on the diffusion of future product line extensions, it was decided to design a single product that would maximize target market acceptance at given expected return.

A survey was performed covering 174 restaurants equipped with microwave ovens or similar equipment. Figure 2 displays the acceptance rates for price given specific product features. Partial acceptance functions are readily obtained by integration, as outlined earlier.

Given the firm's cost structure, an optimization procedure led to the definition of the following product design:

- Frozen shelf life: 3 months

- Meat: Charolais beef

- Vegetables: Dauphinois gratin

- Price: 49FF.

This analysis was performed given current production capacity constraints. Additional analyses are currently underway to develop a line of product offerings and to plan for capacity over the next 5 years. 


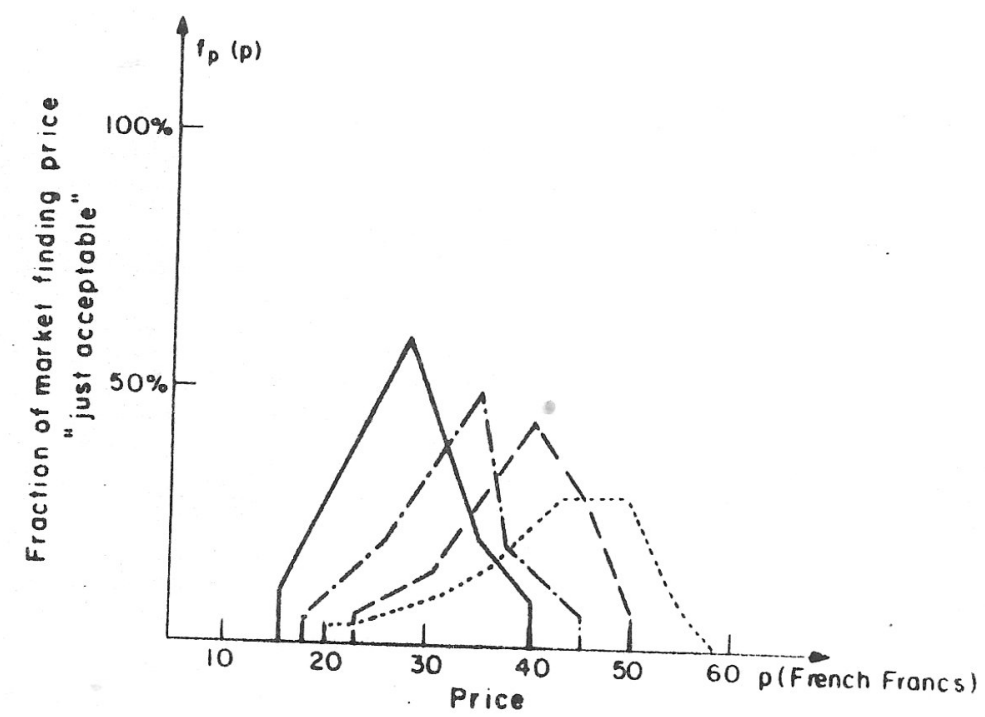

FIGURE 2: Sample acceptance curves (shelf life of 1 month fixed). (---, duck and cre-

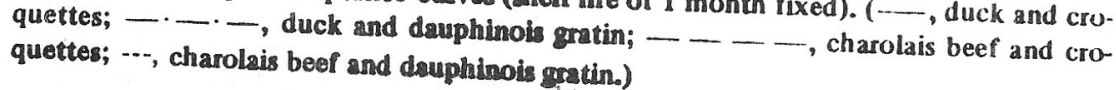

Product Line Extension: Machine Tool The second example is a product manufacturer. The product is a recently for a U.S. industrial preliminary analysis identified thre is a piece of capital equipment and warkary analysis identified three major design dimensions:

- Warranty period (minimum)

- Price per unit of horsepower (maximum)

- Horsepower rating (range).

A survey of the total market potential (comprising about 200 buying organizations) was performed and an acceptability function was
specified as

$$
P(h, w, p)=\alpha_{0}\left[P_{H}(h)\right]^{\alpha_{1}}\left[P_{W}(w)\right]^{\alpha_{2}}\left[P_{D}(d)\right]^{\alpha_{3}}
$$

where

$\boldsymbol{P}_{H}(h)=$ partial market acceptance rate for horsepower rating $P_{w}(w)=$ partial market acceptance rate for warranty period $w$ $P_{D}(d)=$ partial market acceptance rate for price $d$ 
The partial acceptance functions were developed from the empirical distributioms of potential customers requirements using truncated Inormal distributon approximations [2].

Figure 3 illustrates results from the first stage of analysis: design raideofls between warranty length and price per hp. The shape of the isoacceptancie curves suggests that market response is more sensitive to changes in warranty length at low price per hp than at high price. The substitution between these two design dimensions is linear. It also indicates that some acceptance thresholds may exist. For example at $\$ 12.50,34 \%$ of the market finds the product acceptable at a $5 \frac{1 / 2}{2}$-year warranty: further increases in that warranty do not affect market potential estimates.

The DESIGNOR optimization procedure was used to isolate a profitmaximizing product design. It led to the following results:

$$
\begin{aligned}
\text { Maximum Profit } & =\$ 2.61 \mathrm{MM} \text { with design } \\
\text { Price } & =\$ 11.98 / \mathrm{hp} \\
\mathrm{Hp} & =548 \\
\text { Warranty } & =52 / 3 \text { years. }
\end{aligned}
$$

Profit was found to be more sensitive to an increase in price than to a price reduction. A $\$ 1 / \mathrm{hp}$ increase is projected to lead to profit of $\$ 2.19$

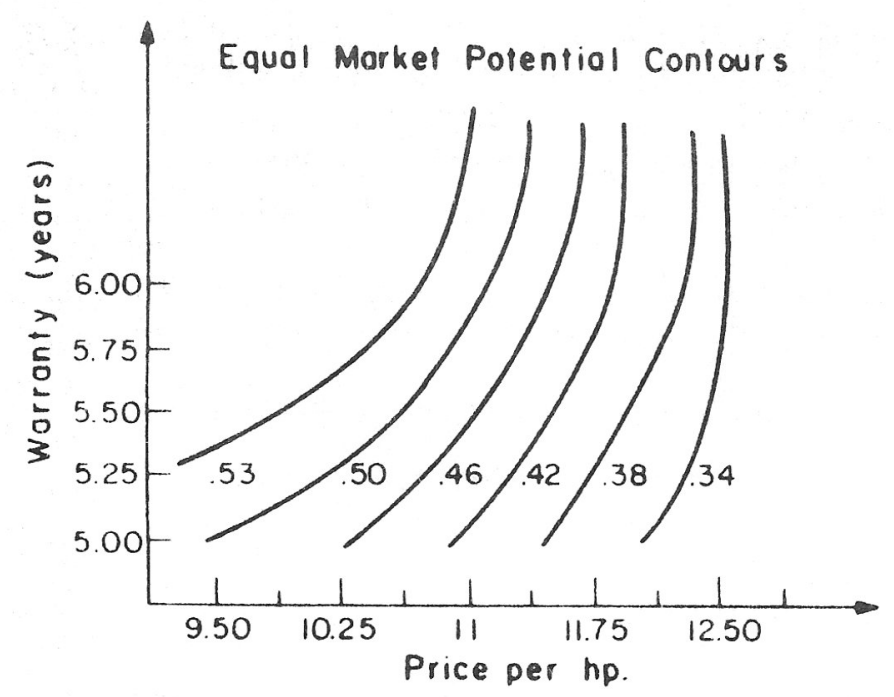

PICIUKI: 3: Market potential tradeoff curves between warranty period and price. 
Table 2: Product Design: A Comparison of Strategies

\begin{tabular}{llcl}
\hline Strategy 1: & One Product & Strategy 2: & Two products \\
\hline Horsepower: & 548 & (1) 537 & (2) 964 \\
Price/hp: & $\$ 11.98$ & $\$ 11.88$ & $\$ 11.88$ \\
Warranty: & 5.66 & 5.66 & 5.66 \\
Profit: & $\$ 2.16 \mathrm{MM}$ & $\$ 3.84 \mathrm{MM}$ & $\$ 3.84 \mathrm{MM}$ \\
\hline
\end{tabular}

MM ( $16 \%$ loss), while a $\$ 1 / \mathrm{hp}$ reduction would lead to profit of $\$ 2.30$ MM ( $10 \%$ loss).

The DESIGNOR model was also used to run more complex sensitivity analyses. One of them concerned the potential benefits of offering a second product with a different horsepower rating, with one product aimed at a market segment with a mean lower horsepower requirement of 400 and the second aimed at a segment with a mean lower horsepower requirement of 800 . In performing this analysis it was assumed that unit sales of products 1 and 2 are equally effective in lowering production cost.

The DESIGNOR optimization procedure led to a profit-maximizing two-product strategy. Table 2 gives the results. The strategy for two products is to design the second one at a horsepower of 963 while lowering the horsepower of the best single-product strategy slightly.

There is an expected profit differential between the two strategies of $\$ 3.84-\$ 2.61=\$ 1.23 \mathrm{MM}$. This suggests to management that, as long as incremental inventory and production and marketing costs are kess than $\$ 1.23 \mathrm{MM} /$ year, a two-product strategy is justified. If these costs are greater than $\$ 1.23$ million, a single-product strategy should be followed. The DESIGNOR methodology thus provides a useful framework for assessing the cost/benefits of various product line extension strattegies.

\section{Discussion and Conclusions}

The model-based methodology presented here focuses on the market potential variation associated with industrial product design alternatives. The output of the analysis is the fraction of the market that would tind a given product design "acceptable." Market demand assessment follows this evaluation as the next step.

A widely discussed method of assessing design tradeoffs for industrial products is conjoint analysis $[14,5]$. The conjoint approach and others discussed by Urban and Hauser [11] provide tools for assessing product 
demand; our position is that these procedures are best applied after a careful market potential assessment, such as that provided by
DESIGINOR.

The approach presented here has been tailored to fit the process of sequential elimination of alternatives, common in industrial markets. Measurement methods that do not impose undue respondent burden are available to calibrate the model.

DESIGNOR is therefore a logical complement to approaches such as conjoint analysis for supporting cost-effective product design decisions. The approach, however, is new and experimental. More needs to be known about the validity of the measurements obtained as well as about the predictive accuracy models.

As presented, the model provides a static view of industrial market response; a dynamic version of the model incorporating temporal adjustments in purchase requirements and diffusion effects is being constructed. That dynamic model will provide a time-varying upper bound on market penetration for diffusion models, blending into the approach suggested by Mahajan and Peterson [8].

\section{References} 1. Anderberg, Michael R., Cluster Analysis for Applications, Academic, New York,

2. Choffray, Jean-Marie, and Lilien, Gary L., DESIGNOR: Decision Support for Industrial Product Design, Sloan School of Management Working Paper, November 1979.

3. Choffray, Jean-Marie, and Lilien, Gary L., Market Planning for New Industrial Products, Wiley, New York, 1980.

4. Crow, Lowell E., Olshavsky, Richard W., and Summers, John O., Industrial Buyers' Choice Strategies: A Protocol Analysis, J. Marketing Res. 17, No. 1 (February 1980): 34-44.

5. I'reehill, M. H., Trade-Off Analysis for Industrial Product Strategy Decisions, in Attitude Research Plays for High Stakes. J. C. Maloney and B. Silverman, eds., American Marketing Association, Chicago, 1979.

6. Kotler, Philip, Marketing Management: Analysis, Planning and Control, 4 th ed., Prentice-Hall, linglewood Cliffs, N.J., 1980.

7. Little, John D. C., Decision Support for Marketing Managers, J. Marketing (Summer 1979): 9-27.

8 Mahajan, Vijay, and Peterson, Robert A., Innovation Diffusion in a Dynamic Potential Adapter Population, Mgt. Sci. (November 1978): 1589-1597.

9. Mansfield, Ldwin, and Wagner, Samuel, Organizational and Strategic Factors Associated with Probabilities of Success in Industrial R\&D, J. Bus. (1975): 179-198. 
10. Tarondeau, J. C., L'Acte d'acht et lo Politique d'Approvisionne'me'nt, 1 is lidition d'Organization, Paris, 1979.

11. Urban, Glen L., and Hauser, John R., Design and Marketing of New Products, Prentice-Hall, Englewood Cliffs, N.J., 1980.

12. Von Hippel, Eric, Successful Industrial Products from Customer Ideas, J. Marke? ing (January 1978): 38-49.

13. Webster, F. E., and Wind, Y., Organizational Buying Behavior, Prentice Hall, Fn glewood Cliffs, N.J., 1972.

14. Wind, Y., Grashof, J. F., and Goldhar, J. D., Market Based Cuidelines ton Design of Industrial Products, J. Marketing 42, No. 3 (1978): 23-37. 